\title{
Alterações morfofuncionais renais em cães Golden Retriever Distróficos (GRMD) ${ }^{1}$
}

\author{
Natalia J.N. Gonçalves²*, Maria A. Miglino², Adriana C. Morini ${ }^{3}$, Daniele S. Martins ${ }^{4}$ \\ e Carlos E. Ambrósio ${ }^{4}$
}

\begin{abstract}
Gonçalves N.J.N., Miglino M.A., Morini A.C., Martins D.S. \& Ambrósio C.E. 2014. [Kidneys morphological changes in Golden Retriever Muscular Dystrophy dogs (GRMD).] Alterações morfofuncionais renais em cães Golden Retriever Distróficos (GRMD). Pesquisa Veterinária Brasileira 34(4):381-384. Departamento de Medicina Veterinária, Faculdade de Zootecnia e Engenharia de Alimentos, Universidade de São Paulo, Av. Duque de Caxias Norte 225, Pirassununga, SP 13635-900, Brazil. E-mail: natgoncalves@usp.br

The Golden Retriever Muscular Dystrophy (GRMD) is genetically homologous to Duchenne muscular dystrophy (DMD) that affects humans. It is a genetic disease that causes progressive degeneration of skeletal muscle. Considering the intense muscle changes, it is natural to think in possible kidney damage caused by intense muscle injury. We evaluated six male Golden Retriever dogs affected by Duchenne muscular dystrophy (GRMD) and three clinically healthy male dogs. The urinary proteins and creatinine concentration were determined. The proteins were analyzed by polyacrylamide gel electrophoresis. The results showed that pathological proteinuria is not directly associated with Duchenne muscular dystrophy, but several parameters showed increased concentrations for affected animals, as the ratio protein/albumin, which was higher in dystrophic dogs, probably a consequence of microalbuminuria a sign of early kidney damage. These results aim to base future studies and clinical evaluations considering the pathologies arising from or associated with this genetic disease.
\end{abstract}

INDEX TERMS: Muscular dystrophy, Golden Retriever, GRMD, renal injury, SDS-PAGE.

RESUMO.- A Golden Retriever Muscular Dystrophy (GRMD) é geneticamente homóloga à distrofia muscular de Duchenne (DMD) que acomete seres humanos. É uma doença genética que gera degeneração progressiva da musculatura esquelética. Considerando-se as intensas alterações musculares, é natural pensar em uma possível lesão renal decorrente da intensa lesão muscular. Foram avaliados seis cães machos da raça Golden Retriever afetados pela distrofia muscular (GRMD) e três cães machos clinicamente sadios. A concentração de creatinina foi determinada e as

\footnotetext{
${ }^{1}$ Recebido em 14 de outubro de 2013.

Aceito para publicação em 24 de março de 2014.

${ }^{2}$ Departamento de Cirurgia, Faculdade de Medicina Veterinária e Zootecnia (FMVZ), Universidade de São Paulo (USP), Av. Prof. Dr. Orlando Marques de Paiva 87, Cidade Universitária, São Paulo, SP 05508-270, Brasil.

${ }^{3}$ Universidade Federal do Oeste do Pará, IBEF - Instituto de Biodiversidade e Florestas. Rua Vera Paz, Maracanã, Santarém, PA 68035110, Brasil

${ }^{4}$ Departamento de Medicina Veterinária, Faculdade de Zootecnia e Engenharia de Alimentos (FZEA), USP, Campus da USP, Avenida Duque de Caxias Norte 225, Pirassununga, SP 13635-900, Brasil. *Autor para correspondência: natgoncalves@usp.br
}

proteínas urinárias foram avaliadas por eletroforese em gel de poliacrilamida. Os resultados mostraram que a proteinúria patológica não está diretamente associada à Distrofia Muscular de Duchenne, porém diversos parâmetros apresentaram concentrações aumentadas para animais afetados, como a razão proteína/albumina, que foi maior em cães distróficos, podendo ser indício de microalbuminúria e conseqüente lesão renal precoce. Estes resultados visam embasar avaliações clínicas e futuros estudos considerando-se as patologias decorrentes ou associadas a esta doença genética.

TERMOS DE INDEXAÇÃO: Distrofia muscular, Golden Retriever, lesão renal, SDS-PAGE.

\section{INTRODUÇÃO}

A Distrofia Muscular de Duchenne (DMD) é a forma mais comum, grave e de evolução mais rápida entre as miopatias hereditárias. É uma doença genética, de caráter recessivo ligado ao cromossomo $\mathrm{X}$, associada a um defeito no gene da distrofina, levando à deficiência ou ausência desta proteí- 
na na membrana da célula muscular. Isto gera degeneração progressiva da musculatura esquelética podendo acometer a musculatura cardíaca e o sistema nervoso (Kornegay et al. 1999).

A Golden Retriever Muscular Dystrophy (GRMD) é geneticamente homóloga à distrofia muscular de Duchenne (DMD) que acomete seres humanos. Sinais clínicos resultantes da ausência da distrofina ocorrem em diversas espécies (Hoffman \& Gorospe 1991), porém em cães, observa-se maior semelhança de evolução da doença com a DMD (Childers et al. 2002, Collins \& Morgan 2003, Bogdonovichi et al. 2004) tendo sido considerados modelos experimentais para diversos estudos que visam à busca de tratamentos para afetados pela doença (Khurana \& Davies 2003).

Os cães afetados são diagnosticados ao primeiro dia de vida, através de teste genético utilizando reação em cadeia de polimerase (PCR) e da análise de creatina quinase, que se encontra elevada na maioria dos casos (Valentine et al. 1990, Childers et al. 2001; 2002).

Considerando-se as intensas alterações musculares e o alto valor da enzima CPK, responsável por catalisar o caminho metabólico creatina-creatinina em células musculares e tecido cerebral, torna-se evidente a possibilidade de lesão renal decorrente da intensa lesão muscular sofrida por esses animais. Os dois parâmetros mais freqüentemente usados para avaliar a função renal são as concentrações plasmáticas de uréia e creatinina (Biewenga \& Van den Bron 1981, Brunker 2005, Grauer 2007).

A quantificação protéica urinária varia amplamente em animais normais e afetados por doenças, refletindo em alterações específicas na estrutura ou função renal. Waller et al. (1989), relataram que a melhor resolução das proteínas urinárias foi obtida pela técnica de SDS-PAGE, as proteínas presentes na amostra se ligam ao SDS na presença de calor, produzindo um íon negativamente carregado que se move através do gel, separando as frações protéicas de acordo com o peso molecular. Lau \& Woo (2002) compararam o exame histopatológico renal com os resultados da eletroforese SDS-PAGE e concluíram que este método pode reduzir a necessidade de biópsia para diagnóstico das afecções renais.

\section{MATERIAL E MÉTODOS}

Foram avaliados de forma comparativa seis cães machos da raça Golden Retriever afetados pela distrofia muscular (GRMD) e três cães machos clinicamente sadios, com idades entre um e quatro anos. Os animais fazem parte do canil GRMD - Brasil, localizado no Departamento de Cirurgia da Faculdade de Medicina Veterinária e Zootecnia da Universidade de São Paulo. O diagnóstico para animais afetados e não afetados foi realizado pelas equipes do Centro de Estudos do Genoma Humano da Universidade de São Paulo (IB-USP) e do Canil GRMD - Brasil, através de genotipagem de acordo com Bartlett et al. (1996).

As amostras de urina foram obtidas quinzenalmente por sondagem uretral, totalizando oito amostras por animal afetado e uma amostra para os animais sadios. A análise física avaliou os parâmetros: cor, aspecto, odor, e densidade. 0 exame químico foi feito com a leitura de padrão de cores da fita reagente Combur 10® (Roche).

As proteínas urinárias foram determinadas utilizando o método colorimétrico do Vermelho de Pirogalol (Kit Sensiprot), a determinação da concentração de creatinina urinária foi baseada na metodologia descrita por Lustgarten \& Wenk (1972), valores da razão proteína/creatinina urinária (RPC) acima de 1,0 foram considerados como proteinúria patológica.

Os estudos eletroforéticos das proteínas foram realizados em sistema vertical de eletroforese em gel de poliacrilamida, com gradiente de $4 \%$ e $10 \%$ de separação, de acordo com o método de preparo de gel descrito por Laemli (1970).

\section{RESULTADOS}

Os resultados da creatinina, uréia, proteína e albumina sérica dos animais afetados apresentaram-se dentro dos padrões (Quadro 1) e não houve diferença significativa quando comparado com os valores apresentados pelos animais normais, de forma geral os valores ficaram dentro da normalidade (valor de referência de $40 \mathrm{mg} / \mathrm{dL}$ do Laboratório Clinico do HoVet-USP) para ambos os grupos. Excetuando-se valores acima do limite estabelecido encontrados em dois animais do grupo afetado (uma amostra do animal I3 e duas dos animais M5 e 2L2). Os valores obtidos de proteína e creatinina urinária foram submetidos à fórmula descrita por Barsanti et al. (2004) (Quadro 1).

Os valores para proteína, creatinina e razão proteína: creatinina urinária (RPC) avaliada no grupo de cães normais apresentaram grande variabilidade entre si, porém mantiveram-se dentro dos níveis de normalidade quando comparados ao grupo de animais afetados (Quadro 2).

Todos os cães nesse estudo apresentaram RPC dentro da normalidade (inferior a 1,0 mg/mL) (Fig.1) mostrando que provavelmente este parâmetro não se altera em decorrência da doença, já em relação as taxas de albumina, o grupo dos cães com distrofia apresentou percentual superior,

Quadro 1. Valores das médias de uréia, creatinina, proteína total e albumina sérica dos cães afetados pela Distrofia Muscular de Duchenne e os cães normais

\begin{tabular}{lcccc}
\hline Animal & $\begin{array}{c}\text { Uréia } \\
\text { Média } \pm \mathrm{s}\end{array}$ & $\begin{array}{c}\text { Creatinina } \\
\text { Média } \pm \mathrm{s}\end{array}$ & $\begin{array}{c}\text { Proteína Total } \\
\text { Média } \pm \mathrm{s}\end{array}$ & $\begin{array}{c}\text { Albumina } \\
\text { Média } \pm \mathrm{s}\end{array}$ \\
\hline 2B7 & $32,3 \pm 3,68$ & $0,71 \pm 0,06$ & $6,27 \pm 0,72$ & $3,2 \pm 0,46$ \\
I3 & $35,05 \pm 11,02$ & $0,76 \pm 0,03$ & $6,5 \pm 0,39$ & $3,05 \pm 0,33$ \\
1K6 & $31,35 \pm 4,78$ & $0,66 \pm 0,02$ & $6,5 \pm 0,55$ & $3,25 \pm 0,37$ \\
2L3 & $36,1 \pm 5,75$ & $0,7 \pm 0,02$ & $7,27 \pm 0,37$ & $3,25 \pm 0,47$ \\
M5 & $41,25 \pm 8,09$ & $0,75 \pm 0,04$ & $6,82 \pm 0,33$ & $3,12 \pm 0,35$ \\
2L2 & $44,42 \pm 11,41$ & $0,79 \pm 0,03$ & $6,8 \pm 0,53$ & $3,3 \pm 0,31$ \\
Normais & $36,76 \pm 2,79$ & $0,59 \pm 0,08$ & $7,53 \pm 0,45$ & 3,1
\end{tabular}

Quadro 2. Valores individuais de proteína, creatinina e razão proteína:creatinina das amostras de urina dos cães do grupo controle e dos cães afetados pela Distrofia Muscular de Duchenne (GRMD)

\begin{tabular}{lccc}
\hline Animal controle & $\begin{array}{c}\text { Proteína } \\
(\mathrm{mg} / \mathrm{mL})\end{array}$ & $\begin{array}{c}\text { Creatinina } \\
(\mathrm{mg} / \mathrm{mL})\end{array}$ & $\begin{array}{c}\mathrm{RPC} \\
(\mathrm{mg} / \mathrm{mL})\end{array}$ \\
\hline Animal 1 & 69,0 & 460,7 & 0,14 \\
Animal 2 & 18,1 & 201,7 & 0,08 \\
Animal 3 & 30,8 & 309,6 & 0,09 \\
Animais afetados & & & \\
2B7 & $84,38 \pm 17,49$ & $359,23 \pm 112,90$ & $0,26 \pm 0,10$ \\
I3 & $47,5 \pm 14,10$ & $260,21 \pm 85,38$ & $0,18 \pm 0,04$ \\
1K6 & $67,26 \pm 24,56$ & $238,95 \pm 53,14$ & $0,29 \pm 0,11$ \\
2L3 & $49,87 \pm 5,28$ & $228,16 \pm 60,13$ & $0,22 \pm 0,04$ \\
M5 & $57,07 \pm 51,39$ & $214,51 \pm 77,51$ & $0,24 \pm 0,12$ \\
2L2 & $97,26 \pm 76,52$ & $265,33 \pm 67,03$ & $0,38 \pm 0,31$
\end{tabular}




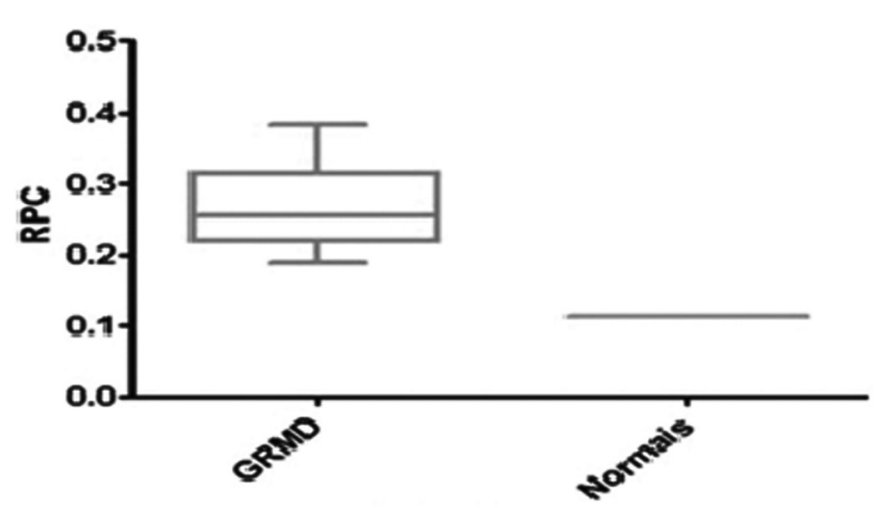

Fig.1. Representação dos valores da mediana (linha horizontal dentro do quadrilátero), percentual de 25\% e 75\% (delimitação d o quadrilátero) e máximo e mínimo (extremidade das barras verticais) da razão proteína:creatinina urinária dos cães afetados pela distrofia (GRMD) e dos cães normais.

porém essa diferença não foi significativa $(\mathrm{p}<0,05)$ (Fig.2).

0 perfil eletroforético das amostras de urina dos cães normais versus afetados apresentaram extrema variabilidade no número de bandas de proteínas e na intensidade óptica de cada banda identificada (Quadro 3).

Foi avaliado o perfil de bandas proteicas para os animais afetados e sadios, apresentando perfis distintos, sendo que os animais estudados não apresentaram proteinúria considerada patológica. Porém apresentaram bandas proteicas de alto peso molecular, um maior percentual de albumina em comparação com os cães normais e um percentual também aumentado de proteínas de baixo peso molecular.

\section{DISCUSSÃO}

Os cães GRMD recebiam uma alimentação com alto valor proteico, além de apresentarem dificuldade na apreensão e deglutição de água e alimentos e intensa sialorréia, promovendo depleção de volume hídrico. A insuficiência renal é uma de várias condições que afetam as concentrações plasmáticas de ureia, portanto o valor de ureia aumentado

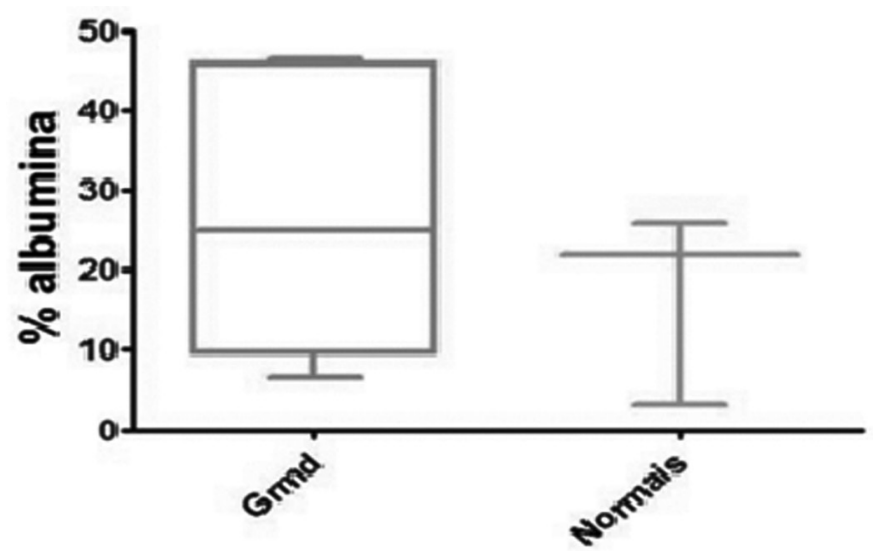

Fig.2. Representação (linha horizontal dentro do quadrilátero), percentis de $25 \%$ e $75 \%$ (delimitação do quadrilátero) e máximo e mínimo (extremidade das barras verticais) da percentagem de albumina na urina dos cães afetados pela distrofia (GRMD) e dos cães normais.
Quadro 3. Análises estatísticas (valor de p) e médias aritméticas da distribuição (\%), das bandas protéicas detectadas em eletroferogramas de urina de cães Goldens Retrievers com Distrofia Muscular de Duchenne (GRMD) e Normais

\begin{tabular}{lccc}
\hline Peso molecular & Grupo GRMD & Grupo normais & Estatística \\
\hline 233 & 0,21 & Ausente & - \\
200 & 1,75 & Ausente & - \\
189 & 1,15 & Ausente & - \\
166 & 0,53 & Ausente & - \\
137 & 1 & Ausente & - \\
114 & 15,71 & 33,9 & $\mathrm{P}=0,54 \mathrm{NS}$ \\
91 & 0,16 & Ausente & - \\
80 & 1,66 & 0,93 & $\mathrm{P}=0,60 \mathrm{NS}$ \\
74 & 1,23 & Ausente & - \\
Albumina & 26,5 & 16,93 & $\mathrm{P}=0,54 \mathrm{NS}$ \\
62 & 3,03 & 4,16 & $\mathrm{P}=0,71 \mathrm{NS}$ \\
57 & 6,08 & 1,5 & $\mathrm{P}=0,09 \mathrm{NS}$ \\
51 & 0,7 & 0,36 & $\mathrm{P}=0,87 \mathrm{NS}$ \\
44 & 2,71 & 0,03 & $\mathrm{P}=0,02 \mathrm{~S}$ \\
36 & 7,51 & 0,16 & $\mathrm{P}=0,02 \mathrm{~S}$ \\
30 & 8,8 & 5,1 & $\mathrm{P}=0,26 \mathrm{NS}$ \\
22 & 10,21 & 1,5 & $\mathrm{P}=0,03 \mathrm{~S}$ \\
18 & 24,11 & 35,4 & $\mathrm{P}=1,00 \mathrm{NS}$ \\
16 & 2,63 & Ausente & -
\end{tabular}

$\overline{\mathrm{NS}}=$ não significativo, $\mathrm{S}$ = significativo.

não é definitivo para o diagnóstico de insuficiência renal, outros exames mais específicos devem ser realizados.

A creatinina se manteve dentro do padrão de referência $(1,3 \mathrm{mg} / \mathrm{dL}$ - Valor de referência do Laboratório Clinico do HoVet-USP), tanto nos animais normais quanto nos cães GRMD. Os valores normais de creatinina sérica geralmente variam de $0,8 \mathrm{mg} / \mathrm{dL}$ a $1,8 \mathrm{mg} / \mathrm{dL}$, dependendo do autor consultado (DiBartola 1997, Barsanti et al. 2004). 0 aumento da creatinina no sangue ocorre em todas as patologias renais em que há diminuição da taxa de filtração glomerular.

Os valores de RPC dos cães do grupo controle foram inferiores a 1,0, valores estes dentro da normalidade, conforme relatos de Lees et al. (2005) e Fettman \& Rebar (2004) que consideram valores acima de 1,0 como proteinúria patológica.

A comparação dos valores de RPC para o grupo controle e para os animais afetados demonstra que as taxas estão significativamente alteradas. De acordo com os estudos de Ortega et al. (1996) e Hurley \& Vaden (1998) relatam que diversas patologias podem ocasionar a perda de proteínas e conseqüente proteinúria patológica.

$\mathrm{Na}$ avaliação individual das bandas de acordo com o peso molecular aproximado das proteínas dos cães pertencentes ao grupo dos cães normais, foram localizadas apenas duas bandas de proteínas de alto peso molecular, a com peso molecular aproximado de 114.000 e 8.0000 dáltons. Zaragoza et al. (2003), observou essas mesmas 2 bandas de alto peso molecular em cães saudáveis e classificou as bandas protéicas encontras em faixas que variam de $10 \mathrm{em}$ 10 , considerando a albumina entre 60 e $70 \mathrm{kDa}$, e de acordo com essa classificação o grupo dos animais desse estudo apresentaram 8 bandas distintas, sendo duas bandas de alto peso molecular, a albumina e outras cinco bandas de peso molecular inferior a albumina. 
O predomínio de proteínas na região correspondente ao peso molecular da albumina foi descrito nos primeiros estudos de eletroforese em gel de poliacrilamida das proteínas urinárias em cães hígidos, sendo que a presença de proteínas de diferentes pesos moleculares na urina de cães sadios é normal, mas em pequenas quantidades (Finco 1995). A proteína de Tamm-Horsfall, proveniente dos rins, é um constituinte normal da urina de cães sadios (Schweigert et al. 2002, Forterre et al. 2004) na sua forma nativa esta proteína é um polímero de peso $>5000 \mathrm{kDa}$, que sofre dissociação em monômeros de peso em torno de 100 $\mathrm{kDa}$ durante a desnaturação por SDS-PAGE (Schweigert et al. 2002). Portanto a banda protéica que aparece na faixa de proteínas de alto peso molecular, não precisa ser necessariamente interpretada como um componente patológico de origem glomerular, mas deve ser visto como um constituinte normal da urina (Raila et al. 2007). A proteína de peso molecular aproximado de $114 \mathrm{kDa}$ que aparece em ambos os grupos pode ser classificada como a proteína de Tamm-Horsfall, que não demonstra nenhuma alteração no presente estudo.

No que se refere à perda de proteína urinária por comprometimento do segmento tubular, esta se caracteriza pelo achado de proteínas de baixo peso molecular $(<60 \mathrm{kDa})$. Os tipos de proteínas presentes na urina de cães normais variam de acordo com o sexo, onde, a presença de proteínas de baixo peso molecular pode estar relacionada à presença de proteínas do trato genital masculino (Rego 2001).

Diante dos dados analisados podemos sugerir que a Distrofia Muscular de Duchenne em cães da raça Golden Retriever, não cursa com proteinúria patológica assim como não se pode afirmar que haja nos cães GRMD lesão renal, tanto pelos exames de rotina, quanto pelos valores de razão proteína:creatinina, tais resultados são coerentes com a pesquisa de Abreu et al. (2012).

Agradecimentos.- Agradecemos ao apoio financeiro da FAPESP para execução da proposta e manutenção da colônia GRMD-Brasil. Processo FAPESP: 2007-51222-2.

\section{REFERÊNCIAS}

Abreu D.K., Costa-de Souza C., Alcântara D., Rodrigues E.A.F., Araújo K.P.C., Maiorka P.C., Miglino M. \& Ambrósio C.E. 2012. Estudo morfofuncional dos rins de cães da raça Golden Retriever afetados pela distrofia muscular (GRMD). Pesq. Vet. Bras. 32:1067-1072.

Barsanti J.A., Lees G.E., Willard M.D. \& Green R.A. 2004. Urinary disorders, p.135-164. In: Willard M.D. \& Tvedten H. (Eds), Small Animal Clinical Diagnosis by Laboratory Methods. ${ }^{\text {th }}$ ed. W.B. Saunders, Missouri.

Bartlett R.J., Winand N.J., Secore S.L., Singer J.T., Fletcher S., Wilton S., Bogan D.J., Metcalf-Bogan J.R., Bartlett W.T., Howell J.M., Cooper B.J. \& Kornegay J.N. 1996. Mutation segregation and rapid carrier detection of X-linked muscular dystrophy in dogs. Am. J. Vet. Res. 57:650-654.

Biewenga W.J. \& Van den Bron W.E. 1981. Assessment of glomerular filtration rate in dogs with renal insufficiency: analyses of the $51 \mathrm{Cr}$-EDTA clearance and its relation to the plasma concentrations of urea and creatinine. Res. Vet. Sci. 30:158-160.

Bogdanovich S., Perkins K.J., Krag T.O. \& Khurana T.S. 2004. Therapeutics for Duchenne Muscular Dystrophy: current approaches and future directions. J. Mol. Med. 82:102-115.

Brunker J. 2005. Protein loosing nephropaty. Comp. Cont. Educ. Pract. Vet. 27:686-695.
Childers M.K., Okamura C.S., Bogan D.J., Bogan J.R., Sullivan M.J. \& Kornegay J.N. 2001. Myofiber injury and regeneration in a canine homologue of Duchenne Muscular Dystrophy. Am. J. Phys. Med. Rehabil. 80:175-181.

Childers M.K., Okamura C.S., Bogan D.J., Bogan J.R., Petroski G.F., McDonald K. \& Kornegay J.N. 2002. Eccentric contractin injury in dystrophic canine muscle. Arch. Phys. Med. Rehabil. 83:1572-1578.

Collins C.A. \& Morgan J.E. 2003. Duchenne's Muscular Dystrophy: Animal Model Used to investigate pathogenesis and develop therapeutic strategies. Int. J. Exp. Pathol. 84:165-172.

Dibartola S.P. 1997. Abordagem clínica e avaliação laboratorial da afecção renal, p.2355-2373. In: Ettinger S.J. \& Feldman E.C. (Eds), Tratado de Medicina Interna Veterinária: moléstias do cão e do gato. $4^{\text {th }}$ ed. Manole, São Paulo.

Fettman M.J. \& Rebar A. 2004. Laboratory evalution of renal function, p.301-328. In: Thrall M.A. (Ed.), Veterinary Hematology and Clinical Chemistry. Williams and Wilkins, Baltimore.

Finco D.R. 1995. Applied physiology of the kidney, p.26-46. In: Osborne C.A. \& Finco D.R. (Eds), Canine and Feline Nephrology and Urology. $2^{\text {nd }}$ ed. Williams and Wilkins, Philadelphia.

Forterre S., Raila J. \& Schweigert F.J. 2004. Protein profiling of urine dogs with renal disease using protein chip analysis. J. Vet. Diagn. Invest. 16:271-277.

Grauer G.F. 2007. Measurement, interpretation, and implications of proteinuria and albuminuria. Vet. Clin. North Am., Small Anim. Pract. 37:283-295.

Hoffman E.P. \& Gorospe J.R.M. 1991. The animal models of Duchenne Muscular Dystrophy: windows on the pathophysiological consequences of dystrophin deficiency. Curr. Trop. Memb. 38:13-154.

Hurley K.J. \& Vaden S.L. 1998. Evaluation of urine protein content in dogs with pituitary-dependent hyperadrenocorticism. J. Am. Vet. Med. Assoc. 3:369-373.

Khurana T.S. \& Davies K.E. 2003. Pharmacological strategies for muscular dystrophy. Nature Rev. 2:379-390.

Kornegay J.N., Bogan D.J., Bogan J.R., Childers M.K., Cundiff D.D., Petroski G.F. \& Schueler R.O. 1999. Contraction force generated by tarsal joint flexion and extension in dogs with Golden Retriever Muscular Dystrophy. J. Neurol. Sci. 166:115-121.

Lustgarten J.A. \& Wen R.E. 1972. Simple, rapid, kinetic method for serum creatinine measurement. Clin. Chem. 8:1419-1422.

Laemli U.K. 1970. Clevage of structural proteins during the assembly of the head of bacteriophage T4. Nature 227:680-685.

Lau Y.K. \& Woo K.T. 2002. SDS-Page in undurutilised as a tool for investigation renal patients. Nephron 90:227-229.

Ortega T.M., Feldman E.C., Nelson R.W., Willits N. \& Cowgill L.D. 1996. Systemic arterial blood pressure and urine protein/creatinina ratio in dogs with hyperadrenocorticism. J. Am. Vet. Med. Assoc. 209:1724-29.

Raila J., Aupperle H., Raila G., Schoon H.A. \& Schweigert F.J. 2007. Renal pathology and urinary protein excretion in a 14-month-old Bernese mountain dog with chronic renal failure. J. Vet. Med. 54:131-135.

Rego A.B., Kogika M.M., Santoro M.L., Hagiwara M.K. \& Mirandola R.M.S. 2001. eletroforese das proteínas urinárias de cães normais e de cães com doença renal em gel de sódio - Dodecil - sulfato poliacrilamida (Sds-Page). Vet. Notícias 7:65-72.

Schweigert F.J., Raila J. \& Haebel S. 2002. Vitamin A excreted in the urine of canines is associated with a Tamm-horsfall like protein. Vet. Res. 33: 299-311.

Valentine B.A., Blue J.T., Sonjia M.S., Shelley D.V.M., Barry J. \& Cooper B.V.S. 1990. Increase serum alanine aminotransferase activity associated with muscle necrosis in the dog. J. Vet. Intern. Med. 4:140-143.

Waller K.V., Ward K.M., Mahan J.D. \& Wismatt D.K. 1989. Current concepts in proteinuria. Clin. Chem. 35:755-765.

Zaragoza C., Barrera R., Centeno F., Tapia J.A., Duran E., Gonzaléz M. \& Mañé M.C. 2003. Sds-Page and western blotting of urinary proteins in dogs with Leishmaniasis. Vet. Res. 34:137-151.

Zaragoza C., Barrera R., Centeno F., Tapia J.A. \& Mañé M.C. 2004. Canine pyometra: a study of the proteins by Sds-Page and western blot. Theriogenology 61:1259-1272. 